A N N A L E S Annales de Bretagne et des Pays de l'Ouest

Anjou. Maine. Poitou-Charente. Touraine

$114-2$ | 2007

Varia

\title{
L'entreprise Noël à Vitré au XX siècle : un chausseur qui réussit
}

Laurence Héry

OpenEdition

Journals

Édition électronique

URL : http://journals.openedition.org/abpo/71

DOI : $10.4000 / a b p o .71$

ISBN : 978-2-7535-1506-2

ISSN : 2108-6443

Éditeur

Presses universitaires de Rennes

Édition imprimée

Date de publication : 30 juin 2007

Pagination : 89-100

ISBN : 978-2-7535-0510-0

ISSN : 0399-0826

Référence électronique

Laurence Héry, "L'entreprise Noël à Vitré au XXe siècle : un chausseur qui réussit », Annales de Bretagne et des Pays de l'Ouest [En ligne], 114-2 | 2007, mis en ligne le 31 décembre 2009, consulté le 19 avril 2019. URL : http://journals.openedition.org/abpo/71 ; DOI : 10.4000/abpo.71 


\title{
L'entreprise Noël à Vitré au $\mathrm{xx}^{\mathrm{e}}$ siècle : un chausseur qui réussit
}

\author{
Laurence HÉRY \\ Doctorante, \\ Groupe d'Histoire des Techniques, LAMOP (UMR 8509) - \\ Université Paris I-Panthéon Sorbonne
}

De l'atelier fondé en 1922 par Henri Noël (associé en 1928 à son frère Édouard), au groupe industriel actuel, quatre-vingts ans s'écoulent : le $\mathrm{XX}^{\mathrm{e}}$ siècle presque entier. Au cours de ce siècle, le fabricant de chaussures vitréen passe d'une toute petite unité à la production de masse, des usines d'Ille-et-Vilaine à la délocalisation puis à un resserrement stratégique. Au cours de ce siècle, une dynastie d'entrepreneurs s'emploie à développer et à faire durer l'activité familiale, de la fulgurante expansion à la désindustrialisation. À ces profondes transformations correspondent des choix stratégiques qui s'effectuent sans que disparaissent certains savoir-faire, veillant à leur transmission et à leur adaptation. Si trois générations de dirigeants ont présidé à ce parcours, des dynasties d'ouvriers et d'employés y font écho. L'entreprise s'est largement appuyée sur un tissu relationnel de proximité, souvent constitué de liens familiaux et en cela, elle peut être considérée comme faisant partie intégrante du patrimoine local.

Largeur de champ, enjeu patrimonial... L'observatoire ne manque pas d'intérêts. Ajoutons, au cœur de la curiosité suscitée par cette entreprise, une anomalie : sa réussite. L'annonce est sciemment provocatrice. Dans l'Entre-deux-guerres, le nombre de fabricants de chaussures en France est estimé à 2000-2500 ${ }^{1}$, puis cette industrie connaît de profondes mutations dans les années cinquante, avant d'être confrontée à un déclin accentué. Ainsi, on ne comptait plus que 199 entreprises qui fabriquaient des chaus-

1. Dans sa thèse (à paraître), Florent Le Bot explique bien la difficulté à retenir un chiffre exact dans la mesure où, en fonction des sources, on ne peut pas savoir si les fabricants de pantoufles sont comptabilisés ou pas. LE Bot, F. « La réaction industrielle. Mouvements antitrust et spoliations antisémites dans la branche du cuir en France, 19301950 ", Université Paris VIII [M. Margairaz], 2004, 3 vol., 786 p. À paraître aux Presses de Sciences Po (automne 2007), sous le titre La Fabrique réactionnaire. Corporatisme, antisémitisme et spoliations dans le monde du cuir en France, 1930-1950. 
sures en France en 2000 (presque 100 millions de paires et 21178 emplois), et 141 en 2004 (53,3 millions de paires et 13380 emplois) ${ }^{2}$. Comment expliquer la réussite de l'entreprise Noël quand d'autres sombrent? Quels sont les ressorts de cette réussite?

Deux axes importants peuvent retenir notre attention pour une ébauche d'explication : le savant dosage de continuité et d'innovation (on pourrait presque dire de continuité dans l'innovation), et l'enracinement dans un territoire.

\section{Une dynastie d'entrepreneurs : continuité et innovation}

"Et comme ça ne cessait pas d'augmenter, les effectifs étaient devenus plus importants et puis les productions étaient aussi... ça progressait. C'était une maison qui ne stationnait pas, ça a toujours toujours progressé e $^{3}$ " " Ici ça bouge tout le temps! L'entreprise et le produit... J'ai jamais éprouvé le besoin d'aller ailleurs ${ }^{4}$. " Les témoignages s'enchaînent parfaitement. Qui ne les a pas recueillis pourrait s'y méprendre. Pourtant, ces deux hommes d'atelier devenus cadres commentent leur carrière à une génération d'intervalle (années soixante et soixante-dix pour le premier, puis années quatre-vingts et quatre-vingt-dix pour le second) et l'on pourrait, pour enrichir et compléter, écouter Urbain Grésil, entré chez Noël en 1930, conter la même histoire en précisant que " ça s'est fait doucement, comme tout ce qui s'est fait chez Noël ${ }^{5}$ ». Contradiction?

\section{Dépasser une apparente contradiction...}

La première occasion qui nous est donnée de constater l'originalité de la " maison Noël » est son fonctionnement complètement contracyclique dans les années trente. La crise économique malmène durement les bassins chaussonniers (Fougères, Romans) quand la production et l'effectif ouvrier augmentent de manière provocante chez Noël à Vitré. Le nombre de paires produites enregistre une poussée très marquée (+ $28 \%$ ) entre 1936 et 1937, probablement liée à l'arrivée de la première chaîne de montage. L'entreprise emploie déjà 125 personnes en 1936 puis 140 à la veille de la guerre. En 1938, elle passe la barre des 100000 paires produites à l'année et, à cette date, $25 \%$ des articles produits sont des chaussures de sport ${ }^{6}$. Au

2. Les Échos, mardi 23 août 2003, p. 1 et p. 16 (d'après des sources fournies par la Fédération française de la chaussure).

3. Entretien du 24 février 2004 avec Monsieur Henri Desbordes, contremaître, responsable d'une chaine de montage chez Noël à partir de 1960.

4. Entretien du 18 février 2002 avec André Orhant, embauché chez Noël en 1975 comme contremaître, directeur de la production depuis trois ans (à la date de l'entretien).

5. Entretien du 2 juillet 2001 avec Monsieur Urbain Grésil, ouvrier chez Noël à partir de 1930 puis contremaître à la coupe des années soixante à 1981 .

6. HÉRY, Laurence, De Noël Frères au Groupe Noël, une entreprise de chaussures à Vitré au XX $X^{e}$ siècle, DEA présenté à l'Université Rennes 2 Haute Bretagne, juin 2003, p. 71-73. 
cours de cette décennie, les frères fondateurs, Henri et Édouard, associés depuis $1928^{7}$, ne se sont pas contentés d'innover en terme de méthode de production. Ils ont aussi fait le choix de diversifier leur fabrication en misant sur le sport, afin d'éviter la difficile période de soudure entre articles d'été et articles d'hiver, période traditionnelle de baisse de l'activité dans l'industrie de la chaussure, et de chômage saisonnier.

Noël Frères est une très jeune entreprise au début des années trente et pour elle, innover, en terme de produit ou de méthode, n'implique pas de profonds changements; cela n'exige pas de renoncer à une manière de faire acquise depuis longtemps. On peut penser que pour les multiples petites unités du Fougerais ou du Romanais, installées dans un tissu artisanal ancien, innover, c'est bouleverser des modes de fonctionnement établis, c'est-à-dire déstructurer. La démarche est difficile quand on est acculé, alors que pour une entreprise comme Noël, qui naît à l'aube de la crise, innover c'est se construire, se structurer et vivre.

Cette attitude, posée par la première génération dans les années trente, imprègne durablement la culture de l'entreprise. "Dans sa marche quotidienne, l'entreprise ne saurait exister sans ajustement constant de sa manière de produire et si possible de vendre ${ }^{8}$. " Au chef d'entreprise, la responsabilité de penser et repenser, compléter et renouveler en continu la réflexion sur le produit, le procès de production, l'organisation de l'entreprise, au chef d'entreprise, aussi, la responsabilité d'évaluer. Si, comme l'écrit Anne-Françoise Garçon, « l'entrepreneur, par définition, est innovant ${ }^{9}$ ", la démarche des frères Noël, dans les années trente, est bien une démarche entrepreneuriale qui inscrit dans la normalité la quête permanente de stratégies qui accroissent la rentabilité, les habitudes d'anticipation et d'innovation.

\section{...Continuité et innovation se marient bien}

Lorsque Xavier Noël, fils d'Édouard et neveu d'Henri, arrive dans l'entreprise en 1955, il entame une action qui va progressivement faire basculer la logique de l'entreprise d'une logique de production vers une logique commerciale. La modernisation s'articule autour d'un triptyque. Il s'agit d'élargir le champ de distribution afin de vendre les produits Noël, non plus dans l'Ouest pour des raisons de proximité ou dans des départements ruraux qui absorbent mieux la production de l'époque, mais dans la France entière, puis dans les pays francophones limitro-

7. Le $1^{\mathrm{er}}$ mars 1928 , les deux frères s'associèrent dans une SARL pour déménager et s'agrandir. Le nouveau statut créé par la loi du 7 mars 1925 constitue l'opportunité de cultiver des ambitions industrielles tout en limitant les risques personnels. En s'installant rue Savary, l'entreprise passe clairement de l'atelier à l'usine.

8. GARÇON, Anne-Françoise, Les Métaux non ferreux en France aux XVIII et XIXe siècles. Ruptures, blocages, évolutions au sein des systèmes techniques, Doctorat d'histoire, Paris, EHESS, 1995, p. 301.

9. Ibidem. 
phes, la sphère coloniale, et rapidement vers les pays de la CEE en train de s'élaborer, en particulier l'Allemagne. Cela passe par l'essor et l'organisation d'un réseau commercial efficace et l'équipe de représentants compte 24 personnes en 1955, puis 30 en 1959, quand ils étaient 11 en $1954^{10}$. Il faut vendre! Mais quoi? Désormais " Noël chausse jeune! " Noël chausse les adversaires dont il faut se méfier! L'entreprise s'appuie sur son savoir-faire ébauché dès avant la guerre dans le domaine du sport mais le choix prend une tout autre ampleur. Une des clefs de la réussite semble être la communication sur le sport " technique " autour de personnages comme Kopa, Albaladejo, Darmon, alors que l'on fabrique et commercialise bientôt massivement, pour l'essentiel, le tennis de trottoir, relativement bon marché, qui fait fortune dans les années soixante-dix. Enfin, en troisième lieu, l'entreprise communique, travaille une image. La réussite économique est spectaculaire. La production court après les ventes et l'entreprise s'agrandit, embauche, sous-traite, essaime en plusieurs ateliers dans le département, délocalise en Tunisie en 1976 tout en continuant d'embaucher en France. En dix ans, entre 1955 et 1965, on est passé d'une entreprise qui avait du mal à écouler sa production à une entreprise qui vend plus de paires de chaussures qu'elle n'en produit, tout en en fabriquant beaucoup plus. La modernisation de l'outil de production et du réseau commercial est allée vite. Une certaine rationalisation de l'espace usinier et le chronométrage ont été mis en place. "C'était une maison qui ne stationnait pas, ça a toujours toujours progressé ", nous a déjà dit Henri Desbordes, contremaître, responsable d'une chaîne de montage dans les années soixante. Les machines arrivent petit à petit, parfois de seconde main, rachetées dans des usines du Fougerais qui ferment. Les adaptations et les améliorations sont courantes, les agrandissements réguliers, les cadences accélérées, les produits, les outils, les marchés peuvent être nouveaux mais d'abords testés par quelques-uns, sans choc frontal. On peut sans doute parler "d'innovation incrémentielle $^{11}$ ", mais de " destruction créatrice " comme le propose Schumpeter, point. De même, la troisième génération, la génération de Patrick, qui prend la relève au début des années quatre-vingt-dix, utilise le statut de société anonyme pour complexifier le groupe, l'organiser autour d'une holding sophistiquée, mener une politique d'achat de licences lucratives (UMBRO) ou de rachat d'entreprises (Minibel, K-Way, Babybotte) dont on fait des filiales. Mais on se spécialise dans la chaussure pour enfant alors que " Noël chausse jeune " depuis les années soixante, et les capitaux sont restés familiaux.

10. HÉRY, Laurence, op. cit., p. 84

11. GARÇON, Anne-Françoise, op. cit., exposé de soutenance, p. 4. Sur ce sujet de l'innovation " incrémentielle " ou " courante ", voir aussi HILAIRE-PÉREz, Liliane, GARÇON, Anne Françoise (dir.), Les Chemins de la nouveauté : innover, inventer au regard de l'histoire, Paris, Éditions du CTHS, 2003, 473 p. 


\section{Défi et mesure : \\ la dialectique permanente de l'entrepreneur chez Noël}

La question de la rupture est donc posée. Quelle figure de l'entrepreneur cultive-t-on chez Noël? Défi et affrontement du risque ou mesure et calcul? "Alexandre, le chevalier entrepreneur " ou " le stratège César " ${ }^{12}$. Faut-il vraiment choisir? Laissons la réflexion d'Hélène Vérin nous éclairer. Elle rappelle que les années quatre-vingt se sont réappropriées « un certain nombre de lieux communs concernant l'entreprise et l'entrepreneur, réactivant des figures de l'entrepreneur que l'on aurait pu croire depuis longtemps révolues ${ }^{13}$ ". Dans la mesure où l'on cherche ce qui fait la différence entre Noël et les autres et où se pose alors, chez Noël, la question de la succession, il ne semble pas illégitime de réutiliser le diptyque qu'elle évoque.

La première figure se caractérise par l'audace et la hardiesse, le goût du défi. En cela, si l'on observe la situation de l'entreprise et de l'industrie de la chaussure aux deux périodes de succession chez Noël, le milieu des années cinquante et le début des années quatre-vingt-dix, on peut dire que la reprise par la génération suivante est un véritable défi. Xavier, en 1955, arrive après deux années de baisse des ventes. Quarante licenciements ont eu lieu en 1954 et il raconte lui-même que son père, Édouard, lui a dit qu'après le droit et HEC, "il fallait peut-être mieux être cadre supérieur dans le pétrole qu'à la tête d'une entreprise de chaussures ${ }^{14}$ ! "

Le défi personnel, familial et économique, est de la même manière, relevé par Patrick, au début des années quatre-vingt-dix, entre deux séries de licenciements traumatisants pour les salariés, alors qu'après l'ESCP, sa propre carrière ne pouvait être ni le seul enjeu ni un motif d'inquiétude. Mais ce défi-là n'a que peu à voir avec le défi chevaleresque remis au goût du jour dans les années quatre-vingt, oublieuses du côté sombre du défi qui comporte en son sein le double risque de la victoire et de la disparition, car c'est bien de durer qu'il s'agit. Hélène Vérin s'interroge : « [...] si entreprendre est commencer, comment y persévérer? " et précise que " dans notre histoire, l'entreprise au sens économique s'est confortée d'un autre modèle : César, le stratège endurant ${ }^{15}$ ». On peut sans doute dire que dans la logique industrielle qui nous occupe, entreprendre c'est justement faire durer l'emprise. La figure du chef d'entreprise doit alors conjuguer en permanence l'audace, le calcul, l'anticipation, l'évaluation. Ces figures de l'entrepreneur ne sont pas exclusives l'une de l'autre et notre observatoire nous en donne l'exemple. Nous avons décrit la deuxième et la troisième génération dans la situation de relever le défi de la succession. Quand

12. VÉRIN, Hélène, "Les figures de l'entrepreneur : histoire et typologie ", dans LAUFER, Romain, HatchuEl, Armand (dir.), Le Libéralisme, l'Innovation et la Question des limites, Paris, L'Harmattan, 2003, p. 17-48.

13. Ibidem, p. 20.

14. Entretien du 25 mai 2001 avec Monsieur Xavier Noël.

15. VÉRIN, Hélène, art. cit., p. 24. 
Xavier Noël délocalise en 1976 et ouvre la première usine tunisienne, il commet un acte pionnier dans l'industrie française de la chaussure et prend un risque dont les retombées sont d'autant moins faciles à envisager qu'il n'y a quasiment pas d'exemples qui permettent la comparaison. Il ouvre une petite unité, éloignée des grands centres urbains alors qu'on lui conseillait le contraire. Cela signifie que l'évaluation du potentiel de cette action a bien eu lieu avant l'action et qu'il se donne le temps d'en évaluer ensuite l'intérêt. Quand, au début des années quatre-vingt, Noël, qui a désormais deux usines tunisiennes, se méfie de "l'après Bourguiba " et ouvre une unité de production au Portugal pour accentuer sa délocalisation, on est pour le moins dans le calcul stratégique et l'anticipation. On pourrait poursuivre en évoquant la troisième génération qui investit dans le rachat d'entreprises, mais seulement après s'être assuré les revenus de l'exploitation de licences commerciales.

De notre observatoire, la figure de l'entrepreneur apparaît ambiguë, cultivant la dualité et mêlant les facettes du diptyque évoqué plus haut. La volonté de saisir ce qui fait l'originalité de l'entreprise Noël - qui s'inscrit dans la durée quand beaucoup d'autres disparaissent - impose maintenant de tenter quelques comparaisons. À partir de la fin des années soixante, les plus grosses entreprises de la chaussure française sont nombreuses à connaître des changements importants de direction, du fait de l'introduction de capitaux extérieurs. À Romans, la prise de contrôle du chausseur Arnoux par le groupe allemand Salamander se fait par étapes entre 1968 et 1973, et se solde par la suppression de 480 emplois ${ }^{16}$ et la disparition d'Arnoux. L'implantation de Salamander à Romans a pour objectif unique l'acquisition d'un réseau de distribution en France à un prix minime; la production, dans cette optique, ne retient plus l'intérêt et aucune logique industrielle n'est à l'œuvre à l'échelle du site romanais " filialisé ». Le " rapport concernant la vérification des services de la Banque à Fougères effectuée en $1968^{17}$ " mentionne que Réhault vient de céder $50 \%$ de son capital au groupe Genesco et que " son orientation n'est pas modifiée dans l'immédiat mais à plus longue échéance, elle sera certainement influencée par les décisions des associés américains ${ }^{18}$ ". L'échéance n'est pas aussi lointaine que semble l'escompter l'observateur de la Banque de France! En 1976, Réhault ferme à Fougères et après 1979, Jourdan, l'ambassadeur du luxe français à l'étranger, dont Genesco, géant américain de la chaussure, est également devenu l'actionnaire majoritaire, entre dans une période assez confuse. On pourrait poursuivre en ajoutant que, dans les années quatre-vingt-dix, les trois chausseurs romanais les plus importants, Jourdan,

16. Les Romanais, Romans et la chaussure, 150 ans d'histoire, (ouvrage collectif, rédaction animée par Jean Sauvageon), ACCES et Édition du Peuple Libre et Notre Temps, 2001, p. 184

17. Archives de la Banque de France, microfilm, bobine 1120, Succursale de Fougères 1946-1968, "rapport concernant la vérification des services de la Banque à Fougères effectuée en 1968 ", vue 332.

18. Idem. 
Clergerie et Kélian, ne sont plus dirigés localement, et allonger la liste. Ces expériences caractérisent une emprise qui n'a pas l'intention de durer. En cela, on pourrait qualifier les prises de contrôle du capital évoquées, d'entreprise chevaleresque, celle-là même qui accepte le risque de la victoire ou de la disparition. En l'occurrence, l'issue n'est pas aussi tranchée qu'on pourrait le croire, ou plus exactement, elle n'est pas la même pour tous, car dans les cas mentionnés, il y a bien rentabilisation des capitaux et perte des emplois, parfois, de la totalité des emplois (Arnoux à Romans, Réhault à Fougères). S'il y a eu des licenciements chez Noël entre 1987 et 1995, et s'ils constituent une cassure dans la mémoire collective des salariés, ils ne font jamais suite à une prise de contrôle de ce type et n'ont pas non plus pour but la fin d'une activité.

\section{D'une génération à l'autre : un passage de témoin préparé et investi}

La comparaison des différentes figures de l'entrepreneur montre une différence fondamentale entre l'entreprise Noël et l'expérience menée par d'autres " grands " (500 à 1200 salariés) de l'industrie de la chaussure en France, depuis 30 à 40 ans, dont beaucoup ont disparu. Cette réflexion ne peut pas être séparée de la question de la formation de l'entrepreneur.

L'exigence que l'activité industrielle s'inscrive dans la durée, exigences de réflexion, de mesure, d'évaluation, nécessitent des savoir-faire et des compétences qui s'acquièrent, une intégration de l'entreprise dans le tissu économique et social local, ainsi que dans une économie à plusieurs échelles. Si on observe le Fougerais, il semble que les directions se caractérisent souvent par " un savoir-faire technique hérité mais non évolutif ${ }^{19}$ ". Chez Noël, on s'assure la complémentarité des compétences et on investit dans la formation de la génération suivante. Si Henri et Édouard étaient bottier et comptable, Xavier sort d'HEC au milieu des années cinquante et Patrick de l'ESCP à la fin des années quatre-vingt. De retour à Vitré, Xavier Noël entreprend des changements fondamentaux et immédiats au sein de l'entreprise familiale, changements qui semblent l'évidence pour un jeune cadre quittant la plus prestigieuse école de commerce de France. Il a en effet reçu une formation hors norme pour un patron de sa génération, dans sa région comme dans son secteur d'activité : la chaussure. Si le décalage est quelque peu atténué pour la troisième génération, il reste une réalité non négligeable. Ainsi, lorsqu'à deux reprises au xxe siècle, se pose la question de la succession, pour des raisons tout autant liées à l'âge du directeur qu'aux réelles difficultés qui malmènent l'industrie de la chaussure en France au milieu des années cinquante et au début des années quatre-vingt-dix, la confiance dans la capacité d'entraînement de la génération suivante, est

19. RÉMY, Allain, "Le système industriel fougerais : de la mono-industrie à la diversification ", dans GESLIN, Claude, La Vie industrielle en Bretagne, une mémoire à conserver, Rennes, PUR, 2001, p. 179. 
un atout majeur. Le passage de témoin se fait sans heurt. La présence de la génération précédente est permanente, physiquement et moralement, mais sans aucun empiétement sur le pouvoir de décision de la nouvelle génération. En dehors de considérations d'ordre privé dont on ne peut pas faire abstraction, cette confiance est facilitée et confortée, y compris parmi les ouvriers, par cette prestigieuse formation. D'une génération à l'autre, cette exigence de formation de la direction apparaît à la fois comme une continuité et une innovation, un gage de renouvellement salutaire, désormais inscrit comme normal dans l'histoire de l'entreprise, mais nullement représentatif de ce qui se fait ailleurs.

\section{Des dynasties d'ouvriers et un territoire}

À ces trois générations d'entrepreneurs font écho des dynasties d'ouvriers et d'employés. L'entreprise est née à Vitré. Elle tire une partie de son originalité du tissu relationnel de proximité (souvent basé sur des relations familiales) qu'elle a élaboré en grandissant au sein de ce territoire, le Pays de Vitré. C'est ce tissu social qui assimile l'innovation courante.

\section{On n'entre pas comme bottier et on ne le devient pas!}

À la première génération, dans les années trente, on quitte l'école pour l'usine sans avoir reçu une quelconque formation.

" [...] Faut bien voir que ceux qui rentraient dans une usine de chaussures à l'époque, c'étaient ceux qui n'avaient pas été à l'école longtemps. On disait... Très clairement les parents disaient aux gosses à l'époque : "si tu travailles pas à l'école, tu iras chez Noël!" Ben oui parce que y avait pas besoin de diplômes, y avait pas d'apprentissage. Si tu voulais être mécanicien, y avait une période d'apprentissage qui n'était pas payée; si tu voulais être menuisier, c'était pareil ${ }^{20}$."

En effet, chez un fabricant de chaussures, toute la formation du jeune s'effectue "sur le tas " et la première heure est payée, "c'était six ou dix sous $^{21}$ ", mais elle était payée. Très souvent, on entre donc chez Noël extrêmement jeune, parfois à la limite de l'âge légal ${ }^{22}$ et sans appartenir à un métier. En un mot, on n'entre pas comme bottier et on ne le devient pas. Presque aucun ouvrier, chez Noël, ni dans les années trente, ni dans les années cinquante, ne sait fabriquer une chaussure. Henri Desbordes est entré chez Noël en 1960. Il avait fait son apprentissage à Vitré dans une autre entreprise et il raconte qu'il fabriquait des chaussures en entier. Il découpait la tige, la cousait lui-même, la montait sur forme. Il faisait tout de A à Z, jusqu'à la finition impeccable; le patron devait se refléter dans

20. Entretien du 2 juillet 2001 avec Monsieur Urbain Grésil, ouvrier chez Noël puis contremaître à la coupe (1930 à 1981).

21. Idem.

22. Urbain Grésil n'a pas encore 12 ans quand il entre chez Noël, un samedi matin d'octobre 1930. 
la chaussure terminée! Quand il est embauché chez Noël en 1960, c'est comme contremaître de chaîne et il est un des seuls à posséder ce savoirfaire de toutes les étapes de la fabrication, donc le seul à pouvoir cultiver une certaine nostalgie, l'idée que "c'était bien parce que au moins quand t'avais créé ce truc-là, tu te disais ben c'est pas mal... C'est pas mal ${ }^{23}$ !" Pour la plupart des ouvriers de chez Noël, la rationalisation des étapes de travail, l'émiettement du travail et son côté de plus en plus répétitif n'impliquent aucun renoncement en terme de savoir-faire. La mécanisation de la coupe correspond à un transfert, à l'acquisition de nouveaux gestes professionnels mais pas à une déqualification. Le banc de piqûre qui relie les machines à coudre et distribue les morceaux de tige à piquer, signifie un changement de rythme, minimise les déplacements mais n'engendre pas de modifications fondamentales du travail de la piqueuse. Au montage, la tâche est répétitive, d'autant plus que les chaînes se multiplient dans les années soixante, mais les gestes professionnels particuliers ou symboliques ne sont pas abandonnés. On a donc à faire à une population ouvrière qui assimile d'autant plus facilement ces innovations en terme de méthode de travail ou de produit, qu'elles n'impliquent pas, le reniement d'un métier porteur d'identité. Dans les années soixante et soixante-dix, le fait d'appartenir à une entreprise qui produit, vend, embauche de plus en plus, est un motif de satisfaction.

\section{Un monde ouvrier sans habitudes revendicatives}

La cristallisation de l'opposition à ces innovations aurait alors pu se faire sur les conditions de travail et de salaires. Non sur l'arrivée de la machine, mais sur le nouveau rendement exigé. Non sur l'émiettement de la fabrication du produit mais sur la cadence imposée. C'est parfois le cas ailleurs, mais pas à Vitré. L'arrondissement n'a pas d'habitudes d'organisation ouvrière et de revendication syndicale. En 1936, les voix du PCF et de la SFIO, au premier tour des élections législatives, représentent 20 à $30 \%$ des inscrits dans l'arrondissement de Fougères mais moins de $10 \%$ des inscrits dans celui de Vitré ${ }^{24}$.

"Ainsi, alors que le Front populaire triomphe dans la plus grande partie du Pays, dans notre département sont élus des députés modérés n'appartenant pas au Front populaire, voire franchement hostiles, mais les candidats de l'extrême droite fascinante sont aussi écartés. Dans l'arrondissement de Vitré, Jean Bohuon, lieutenant de Dorgères, est battu par le candidat conservateur, et, cela, malgré le rapide développement des comités de défense paysanne ${ }^{25}$."

23. Entretien du 24 février 2004 avec Monsieur Henri Desbordes, contremaître, responsable d'une chaîne de montage chez Noël à partir de 1960.

24. LEBRUN, François (dir.), L'Ille-et-Vilaine des origines à nos jours, Saint-Jean-d'Angély, Éditions Bordessoules, 1984, carte p. 415.

25. Ibidem, p. 416 . 
Si l'on peut apercevoir le mécontentement, aux relents fascistes, d'une paysannerie qui a subi de plein fouet la crise des années trente, on est très loin d'une ville qui verrait se structurer une organisation du monde ouvrier. Le calme social reste une caractéristique inchangée du tissu vitréen. $\mathrm{Si}$ la vie syndicale est plus ouverte chez Noël avec l'arrivée de la deuxième génération, la CGT, qui ne parvient pas à trouver un ancrage, fait un passage éclair et sans trace. FO apparaît relativement tardivement dans les années soixante-dix, quand l'entreprise a beaucoup grossi. La CFTC, devenue CFDT, occupe le premier plan depuis 1964. Pour l'inspectrice du travail qui a visité l'entreprise pendant 30 ans (à partir de 1970), il y a une vie syndicale et une liberté de ton chez Noël qui n'existent nulle part ailleurs dans les entreprises vitréennes. Mais ceci reflète surtout une certaine habileté de la direction car laisser dire c'est savoir anticiper et parfois désamorcer les conflits. Quand l'entreprise grossit, dans les années soixante-dix, elle essaime en plusieurs petites usines très spécialisées autour de Vitré, à Liffré, Renazé, La Guerche. Ce qui pourrait apparaître comme peu judicieux, puisque consommateur de temps et de transport, comporte des atouts en terme de productivité, et constitue également un outil de contrôle efficace, d'une main-d'œuvre qui peine toujours à s'organiser dans des unités éclatées. D'une certaine manière, dans l'expérience tunisienne qui commence en 1976, le refus de construire une grosse usine dès le départ dans un grand centre urbain peut être compris dans la continuité des relations mises en place avec les ouvriers vitréens.

Les conditions de travail sont difficiles chez Noël mais sans doute finalement ni plus ni moins que dans d'autres entreprises vitréennes ou dans ces usines de la " décentralisation " qui s'implantent pour exploiter le filon de la main-d'œuvre abondante, endurante aux travaux physiquement exigeants, peu revendicative, d'autant moins d'ailleurs qu'elle aspire à " vivre et travailler au pays ${ }^{26}{ }$. Cette main-d'œuvre est bien celle du bassin d'emploi vitréen, celle que l'entreprise Noël embauche et utilise comme un avantage comparatif dans les années soixante et soixante-dix, cette main-d'œuvre avec laquelle les dirigeants ont tissé des liens ténus, notamment par le biais d'une " gestion sociale " originale.

\section{Amortisseur social et concordat}

L'entreprise n'a pas toujours été un modèle sur le plan " hygiène et sécurité ". La gestion comptable au plus près et la chasse au gaspillage, caractéristiques du fonctionnement de la " maison Noël » jusqu'à la fin du Xxe siècle, n'ont pas toujours contribué à résoudre de la façon la plus

26. Le slogan de l'UDB, très largement repris par la CFDT, fait florès. Il correspond sans doute à une aspiration réelle et en même temps, cette aspiration se fait d'autant plus prégnante que le slogan est bien relayé. La CFDT est active chez Noël à Vitré, dans les années soixante-dix, quand elle relaye ce slogan. L'aspiration entretenue incite au concordat, si cela était nécessaire, une population ouvrière qui n'a aucune habitude revendicative et semble adhérer à la gestion sociale de la direction. 
confortable ou la plus rapide des problèmes concrets, posés, par exemple, par l'exiguïté des locaux (récurrente dans une entreprise dont la taille ne cesse d'augmenter). Les phalanges tronquées, les douleurs du canal carpien, les odeurs de colle sont aussi là pour rappeler que l'on n'a pas à faire à des métiers sans risque dans une entreprise qui fabrique des chaussures. Mais en même temps, le " comité social " créé en 1942 conformément aux exigences de Vichy, est transformé, en 1951, en une "société mutualiste" qui adhère à une caisse de retraite complémentaire dès $1959^{27}$. Face à des conditions de travail difficiles et à des petits salaires, l'amortisseur social que constitue la mutuelle est un facteur efficace de paix sociale et de stabilisation de la main-d'œuvre. Les ouvriers entrent jeunes chez Noël. Certains $\mathrm{y}$ font toute leur carrière, parfois avec d'autres membres de leur famille, et la plupart des personnes qui témoignent de leur vie professionnelle dans cette entreprise disent n'avoir pas songé à aller ailleurs, à part au milieu des années cinquante et au début des années quatre-vingt-dix, quand pouvait se poser la question du chômage.

La mutuelle n'est pas le seul outil de "gestion sociale " qui soit à la fois au service de la stabilité et de la productivité. La promotion interne, pratiquée de manière systématique, constitue le moyen de s'attacher des fidélités en répondant à des aspirations d'avancement personnel. Le recrutement se fait très peu à l'extérieur du bassin d'emploi local, même pour les cadres qui sont le plus souvent des ouvriers ou employés " choisis ". Ils sont envoyés en formation à Romans où sont dispensées des formations techniques spécifiques aux besoins de l'industrie de la chaussure, mais ils appartiennent au sérail et possèdent la culture de l'entreprise. De Noël Frères au Groupe Noël, l'entreprise s'enracine bel et bien dans un territoire dont elle puise, sur le plan humain, des ressources collectives sans abandonner le jeu des relations individualisées.

Noël, fabricant de chaussures à Vitré, est une entreprise qui s'inscrit dans la durée, ou pour être précis, dans une durée beaucoup plus longue que celle de la plupart des chausseurs français qui sont devenus aujourd'hui fort rares. On a effleuré l'idée que la réussite pouvait être relative et diverger en fonction des acteurs observés mais le terme est sans doute adapté pour Noël. Expliquer les ressorts de cette réussite impliquerait des chantiers nombreux et diversifiés qu'il n'était pas envisageable d'ouvrir ici.

Pour ce qui est de la longue durée, les deux axes qui ont retenu notre attention montrent des originalités éclairantes concernant le fonctionnement de la " maison Noël ". En effet, la façon dont une dynastie d'entrepreneurs inscrit en continu l'innovation comme une normalité, avec ce que cela implique d'évaluation du risque, de dosage entre défi et calcul, de formation de l'entrepreneur, n'est pas représentatif de choix qui ont pu se faire ailleurs dans ce secteur d'activité et peut constituer un élément

\footnotetext{
27. La société mutualiste de l'entreprise est absorbée avec tous ses membres par la
} « mutuelle des marches de Bretagne " en 1987. 
d'explication de la réussite. Il faut cependant rappeler que la troisième génération a fait le choix d'une orientation de plus en plus commerciale et qu'il est légitime de se demander si l'avenir verra le Groupe Noël conserver son ancrage industriel. Enfin, ni le piètre attachement à un métier, ni la faible qualification de la majorité des salariés, ni l'absence d'habitudes politiques et syndicales revendicatives, ni la grande habileté d'un patronat rompu à l'utilisation de l'outil social et vigilant à limiter les possibilités d'organisation ouvrière ne sont introuvables ailleurs. C'est sans doute la combinaison de ces facteurs qui doit être approfondie parce qu'elle est plus originale. Elle fait apparaître la longévité comme indissociable du territoire dans lequel l'entreprise s'enracine et du tissu social qui le caractérise.

\section{RÉSUMÉ}

Au Xx ${ }^{\mathrm{e}}$ siècle, l'histoire de l'entreprise Noël à Vitré (Ille-et-Vilaine) est l'histoire d'un chausseur qui gagne. Si la longue vie de cette entreprise familiale fait d'elle un objet d'étude particulièrement riche, Noël suscite surtout la curiosité parce qu'elle " réussit ", alors qu'en France, l'industrie de la chaussure connaît un déclin accentué.

Expliquer en quelques pages l'ensemble des ressorts de cette réussite serait une gageure. Mais on tentera de suivre ici deux pistes éclairantes : l'alliance entre continuité et innovation ainsi que le lien puissant à un territoire et aux hommes qui le constituent.

\section{ABSTRACT}

In the twentieth century, Noël enterprise's story in Vitré (Ille-et-Vilaine, Brittany) is a winner's story in the shoe industry. Its long life makes of Noël a large and interesting matter. But most of all, for the researcher, Noël is appealing because this enterprise succeed when the shoe industry collapses in France. It would be attempting the impossible to explain this success story in a few pages. We'll try then to follow there two enlightening paths: the union of continuity and innovation and the close bond between this enterprise and an area. 